\title{
An Improved Spatial Difference Smoothing Method Based on Multistage Wiener Filtering
}

\author{
Jian Gong $\mathbb{D}^{1,2,3}$ Yiduo Guo $\mathbb{D}^{2},{ }^{2}$ and Qun Wan ${ }^{1}$ \\ ${ }^{1}$ School of Communication and Information Engineering, University of Electronic Science and Technology of China, Chengdu, \\ Sichuan, China \\ ${ }^{2}$ Air and Missile Defense College, Air Force Engineering University, Xi'an, Shaanxi, China \\ ${ }^{3}$ Tong Fang Electronic Science and Technology Co. Ltd., Jiujiang, Jiangxi 332007, China \\ Correspondence should be addressed to Yiduo Guo; guoyiduo111@126.com
}

Received 27 April 2019; Revised 15 July 2019; Accepted 9 October 2019; Published 7 November 2019

Academic Editor: Filippo Cacace

Copyright (C) 2019 Jian Gong et al. This is an open access article distributed under the Creative Commons Attribution License, which permits unrestricted use, distribution, and reproduction in any medium, provided the original work is properly cited.

\begin{abstract}
In order to solve the angle estimation problem of coherent sources in the colored background noise, an improved forward and backward spatial difference smoothing algorithm is proposed by combining the improved spatial smoothing algorithm with the spatial difference algorithm. By the algorithm we can not only decoherent the coherent source but also suppress the influence of the color noise. In order to further reduce the computational complexity of the IFBSDS algorithm, an improved forward and backward spatial difference smoothing algorithm based on Wiener filtering is also proposed. Thus, the eigenvalue decomposition operation of subspace class algorithm can be avoided, and at the same time, the same performance with the IFBSDS algorithm can be obtained, which is more consistent with the real demand of MIMO radar signal real-time processing.
\end{abstract}

\section{Introduction}

At present, the study of MIMO radar target angle estimation generally assumes that the environmental noise is white noise obeying Gauss distribution [1-3]. However, in practical applications, there are many factors such as the electronic interference caused by the enemy, the random scattering caused by distributed source, and the mutual coupling characteristics between the receiving channels and so on, and ambient noise often appears as a nonideal colored noise with unknown statistical characteristics. Besides, electronic jamming and low-altitude multipath effect make MIMO radar need angle estimation for coherent sources [4-6]. Under the condition of colored noise background and coherent source, the performance of the classical subspace algorithm such as multiple signal classification (MUSIC) algorithm and estimating signal parameter via rotational invariance techniques (ESPRITs) algorithm will become very poor [7-9]. The four-order cumulant matrix of echo data is constructed in $[10,11]$, and the angle of the target can be estimated effectively, under the background of colored noise and white noise, but the influence of coherent sources is not eliminated. In [12], an extended rotation invariance factor is obtained for the implementation of the four-order cumulative data, which has high aperture utilization and no phase ambiguity, but the computational complexity is high. In $[13,14]$, the covariance matrix is reconstructed through the Toeplitz submatrix of a set of received data, but the Gauss color noise cannot be suppressed. In [15], an improved spatial differencing method (ISD) is proposed by constructing a spatial differencing matrix with neighboring subarrays, and the proposed method performs better under the coexistence of both uncorrelated and coherent signals. In [16], by extracting all the data information of each subarray, both the forward only ISD (FOISD) and forward backward ISD (FB-ISD) methods are proposed using the reconstructed submatrices.

In this paper, an improved spatial difference smoothing algorithm based on multistage Wiener filtering is proposed. The remainder of this paper is organized as follows. In Section 2, the echo model is established and its characteristics under color noise are analyzed. Then, an improved spatial difference smoothing (ISDS) algorithm based on 
multistage Wiener filtering is proposed in Section 3, followed by performance analysis of ISDS algorithm based on multistage Wiener filtering (MWF-ISDS) in Section 4. Some simulations are conducted to verify the performance of the proposed method in Section 5. Finally, we conclude the paper in Section 6.

Notation: $(.)^{*}$ denotes the conjugate operator; $(.)^{\mathrm{H}}$ denotes the matrix conjugate-transpose operator; $(.)^{\mathrm{T}}$ denotes the matrix transpose operator; $\otimes$ denotes the Kronecker product operator; $\Sigma$ denotes the sum operator; $\Pi$ denotes the multiplication operator.

\section{Echo Model and Its Characteristics under Color Noise}

Considering the single base MIMO radar as shown in Figure 1, the transmitting and receiving antenna adopt $M$ and $N$ elements, respectively. The spacing of array elements is $d=\lambda / 2$, and $\lambda$ is the carrier wavelength. Assuming that the direction of arrival (DOA) and the direction of departure (DOD) both are $\theta_{\mathrm{p}}$, the echo signal is processed by matching filtering as below:

$$
\mathbf{y}=\mathbf{A} \boldsymbol{\beta}(t)+\mathbf{n}(t),
$$

where $\quad \mathbf{A}=\left[\mathbf{a}_{\mathrm{r}}\left(\theta_{1}\right) \otimes \mathbf{a}_{\mathrm{t}}\left(\theta_{1}\right), \mathbf{a}_{\mathrm{r}}\left(\theta_{2}\right) \otimes \mathbf{a}_{\mathrm{t}}\left(\theta_{2}\right), \ldots, \mathbf{a}_{\mathrm{r}}\left(\theta_{\mathrm{P}}\right) \otimes \mathbf{a}_{\mathrm{t}}\right.$ $\left.\left(\theta_{\mathrm{P}}\right)\right]$, the transmit steering vector of the pth target is $\mathbf{a}_{\mathrm{t}}\left(\theta_{\mathrm{p}}\right)=\left[1, \exp \left(-j \pi \sin \theta_{\mathrm{p}}\right), \ldots, \exp \left(-j \pi(M-1) \sin \theta_{\mathrm{p}}\right)\right]^{\mathrm{T}}$, the receive steering vector is $\mathbf{a}_{\mathrm{r}}\left(\theta_{\mathrm{p}}\right)=[1, \exp (-j \pi$ $\left.\left.\sin \theta_{\mathrm{p}}\right), \ldots, \exp \left(-j \pi(N-1) \sin \theta_{\mathrm{p}}\right)\right]^{\mathrm{T}}, \quad \boldsymbol{\beta}=\left[\xi_{1} e^{j 2 \pi f_{\mathrm{d} 1} t}, \xi_{2}\right.$ $\left.e^{j 2 \pi f_{\mathrm{d} 2} t}, \ldots, \xi_{\mathrm{p}} e^{j 2 \pi f_{\mathrm{dP}} t}\right]^{\mathrm{T}}, \xi_{\mathrm{p}}$ is the reflection coefficient of the pth target, $f_{\mathrm{dp}}$ is the normalized Doppler frequency of the pth target, and $\mathbf{n}(t)$ representation of noisy column vectors.

If defined,

$$
\mathbf{a}_{\mathrm{r}}\left(\theta_{\mathrm{p}}\right) \otimes \mathbf{a}_{\mathrm{t}}\left(\theta_{\mathrm{p}}\right) \triangleq \mathbf{F} \mathbf{b}\left(\theta_{\mathrm{p}}\right) .
$$

In the form, $\mathbf{b}\left(\theta_{\mathrm{p}}\right)=\left[1, \exp \left(-j \pi \sin \theta_{\mathrm{p}}\right), \ldots, \exp (-j \pi\right.$ $\left.\left.(M+N-1) \sin \theta_{\mathrm{p}}\right)\right]^{\mathrm{T}}, \mathbf{F} \in \mathbf{C}^{M N \times(M+N-1)}$ is a dimensionality reduction matrix, which can be expressed as [18]

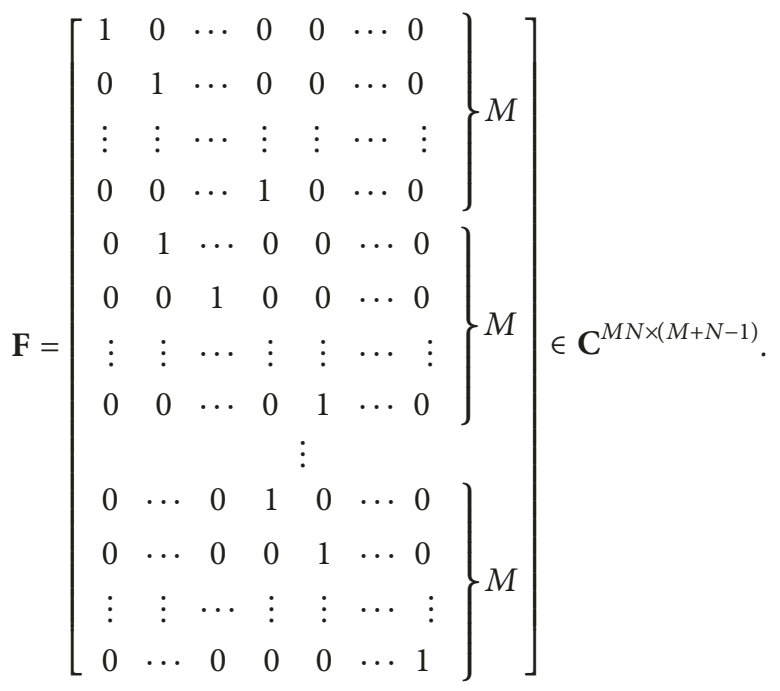

Then, the matrix $\mathbf{A}$ can be expressed as follows:

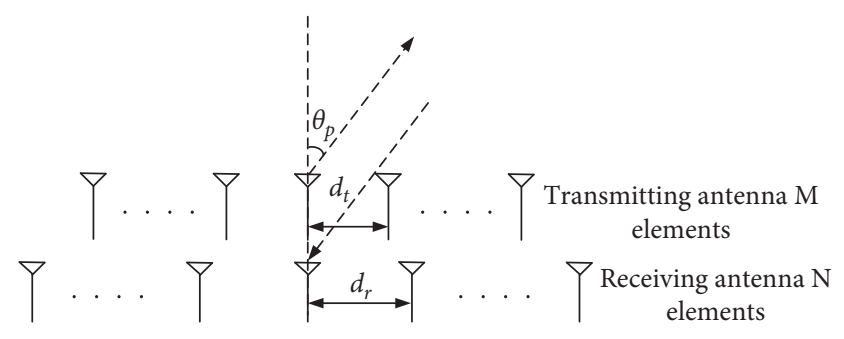

- pth target

FIgURE 1: The structure of a monostatic MIMO radar [17].

$$
\mathbf{A}=\mathbf{F B},
$$

where $\mathbf{B}=\left[\mathbf{b}\left(\theta_{1}\right), \mathbf{b}\left(\theta_{2}\right), \ldots, \mathbf{b}\left(\theta_{\mathrm{P}}\right)\right] \in \mathbf{C}^{(M+N-1) \mathrm{P}}$, and it is a Vandermonde matrix.

According to formula (3), if the formula $\mathbf{W} \triangleq \mathbf{F}^{\mathrm{H}} \mathbf{F}$ is defined, there are

$$
\mathbf{W}=\operatorname{diag}(1,2, \ldots, \underbrace{\min (M, N), \ldots, \min (M, N)}_{|M-N|+1}, \ldots, 2,1) .
$$

By using the reduced dimension transformation $\mathbf{W}^{-1} \mathbf{F}^{\mathrm{H}}$, we can get

$$
\begin{aligned}
\mathbf{y}^{\prime}\left(t_{1}\right) & =\mathbf{W}^{-1} \mathbf{F}^{\mathrm{H}} \mathbf{y}\left(t_{1}\right)=\mathbf{W}^{-1} \mathbf{F}^{\mathrm{H}}(\mathbf{F B}) \boldsymbol{\beta}\left(t_{1}\right)+\mathbf{W}^{-1} \mathbf{F}^{\mathrm{H}} \mathbf{n}\left(t_{1}\right) \\
& =\mathbf{B} \boldsymbol{\beta}\left(t_{1}\right)+\mathbf{n}^{\prime}\left(t_{1}\right),
\end{aligned}
$$

where $\mathbf{n}^{\prime}\left(t_{1}\right)=\mathbf{W}^{-1} \mathbf{F}^{\mathrm{H}} \mathbf{n}\left(t_{1}\right)$ is a $(M+N-1)$ dimension additive noise vector.

When there are independent sources and coherent sources in the $P$ target, it may be possible to set up $G$ as coherent source number, and then $U=P-G$ is the number of independent source. The array manifold composed of coherent sources can be expressed as $\mathbf{B}_{\mathbf{c}}=\left[\mathbf{b}\left(\theta_{1}\right), \ldots, \mathbf{b}\left(\theta_{\mathrm{G}}\right)\right]$, and the array manifold composed of independent sources can be represented as $\mathbf{B}_{\mathrm{nc}}=\left[\mathbf{b}\left(\theta_{G+1}\right), \ldots, \mathbf{b}\left(\theta_{\mathrm{P}}\right)\right]$. Incoherent sources can be expressed as $\boldsymbol{\beta}_{\mathrm{nc}}(t)=\left[\beta_{\mathrm{G}+1}(t), \ldots, \beta_{\mathrm{P}}(t)\right]^{T}$. If $\rho_{i}$ is used to represent the generation factor of the ith coherent source, the coherent source can be expressed as $\boldsymbol{\beta}_{\mathrm{c}}(t)=\rho \beta_{0}(t)$, the echo signal of the matched filter can be expressed as follows:

$$
\begin{aligned}
\mathbf{y} & =\sum_{i=1}^{G} \mathbf{b}\left(\theta_{i}\right) \rho_{i} \beta_{0}(t)+\sum_{j=G+1}^{P} \mathbf{b}\left(\theta_{j}\right) \beta_{j}(t)+\mathbf{n}^{\prime}(t) \\
& =\mathbf{B}_{\mathrm{c}} \boldsymbol{\beta}_{\mathbf{c}}(t)+\mathbf{B}_{\mathrm{nc}} \boldsymbol{\beta}_{\mathrm{nc}}(t)+\mathbf{n}^{\prime}(t) .
\end{aligned}
$$

So, the covariance matrix $\mathbf{R}$ is

$$
\begin{aligned}
\mathbf{R} & =\mathbf{B}_{\mathrm{c}} \mathbf{P}_{\mathrm{c}} \mathbf{B}_{\mathrm{c}}^{\mathrm{H}}+\mathbf{B}_{\mathrm{nc}} \mathbf{P}_{\mathrm{nc}} \mathbf{B}_{\mathrm{nc}}^{\mathrm{H}}+\boldsymbol{\varphi} \\
& =\mathbf{R}_{\mathrm{c}}+\mathbf{R}_{\mathrm{nc}}+\boldsymbol{\varphi},
\end{aligned}
$$

where $\varphi$ represents the color noise covariance matrix and $\mathbf{R}_{c}$ and $\mathbf{R}_{\mathrm{nc}}$ are the covariance matrix of the coherent source and the independent source, respectively. Among them, $\mathbf{R}_{\mathrm{nc}}$ is Hermite, Toeplitz matrix, $\mathbf{R}_{c}$ is Hermite, non-Toeplitz matrix, and $\varphi$ is Hermite, Toeplitz matrix when space stationary color noise. 
Under the color noise, there is a large angle estimation error in the classical DOA algorithm. The angle estimation loss caused by the theoretical noise than the real noise can be found in the literature [19].

\section{ISDS Angle Estimation Algorithm Based on Multistage Wiener Filtering}

3.1. Improvement of Spatial Smoothing Algorithm. Assuming that the number of virtual elements after the dimension reduction transformation is $I$, then $I=M+$ $N-1$. The forward space smoothing algorithm divides the array into $q(q \geq G+1)$ subarrays. The number of array elements of each subarray is $m=I-q+1$. By using the translation invariance property between subarrays, the covariance matrix of $q$ subarrays can be expressed as follows:

$$
\mathbf{R}_{m}^{i i}=\mathbf{B}_{m} \mathbf{D}^{i-1} \mathbf{P}\left(\mathbf{D}^{i-1}\right)^{\mathrm{H}} \mathbf{B}_{m}^{\mathrm{H}}+\sigma^{2} \mathbf{I}_{m}, \quad i=1, \ldots, q,
$$

where $\mathbf{R}_{m}^{i i}=\mathbf{R}(i: I+i-q, i: I+i-q), \quad \mathbf{B}_{m}$ is an array manifold of the initial subarray, $\sigma^{2}$ indicates noise power, and $\mathbf{D}=\operatorname{diag}\left[e^{-j \pi \sin \theta_{1}}, e^{-j \pi \sin \theta_{2}}, \ldots, e^{-j \pi \sin \theta_{\mathrm{G}}}\right]$.

The equivalent covariance matrix $\mathbf{R}_{\mathrm{f}}$ of the conventional forward space smoothing algorithm can be obtained as follows:

$$
\mathbf{R}_{\mathrm{f}}=\frac{1}{q} \sum_{i=1}^{q} \mathbf{R}_{m}^{i i}
$$

And the covariance matrix of the forward and backward spatial smoothing subarray can be expressed as follows:

$$
\mathbf{R}_{\mathrm{fb}}=\frac{1}{2 q} \sum_{i=1}^{q}\left[\mathbf{R}_{m}^{i i}+\bar{R}_{m}^{i i}\right]=\frac{1}{2 q} \sum_{i=1}^{q}\left[\mathbf{R}_{m}^{i i}+\mathbf{J}\left(\mathbf{R}_{m}^{i i}\right)^{*} \mathbf{J}\right]
$$

where $\mathbf{J}$ represents the $m$ order permutation matrix and * represents the conjugate operation.

In order to further excavate the useful information of the received data, this paper improves the conventional spatial smoothing algorithm from two directions: strengthening the influence of the main diagonal elements and adding intercorrelation information between the subarrays. The new smoothing matrix performs cross-correlation operations on the $q$ subarray autocorrelation matrix, and then the crosscorrelation matrix of the symmetric subarray is cross-correlation to obtain the average as follows:

$$
\begin{aligned}
\widetilde{\mathbf{R}}_{f} & =\frac{1}{2 q} \sum_{i=1}^{q} \sum_{j=1}^{q}\left(\mathbf{R}_{m}^{i i} \mathbf{R}_{m}^{j j}+\mathbf{R}_{m}^{i j} \mathbf{R}_{m}^{j i}\right), \\
\widetilde{\mathbf{R}}_{\mathrm{fb}} & =\frac{1}{4 q} \sum_{i=1}^{q} \sum_{j=1}^{q}\left[\left(\mathbf{R}_{m}^{i i} \mathbf{R}_{m}^{j j}+\mathbf{R}_{m}^{i j} \mathbf{R}_{m}^{j i}\right)+\left(\overline{\mathbf{R}}_{m}^{i i} \overline{\mathbf{R}}_{m}^{j j}+\overline{\mathbf{R}}_{m}^{i j} \overline{\mathbf{R}}_{m}^{j i}\right)\right] \\
& =\frac{1}{4 q} \sum_{i=1}^{q} \sum_{j=1}^{q}\left[\left(\mathbf{R}_{m}^{i i} \mathbf{R}_{m}^{j j}+\mathbf{R}_{m}^{i j} \mathbf{R}_{m}^{j i}\right)+\mathbf{J}\left(\mathbf{R}_{m}^{i i} \mathbf{R}_{m}^{j j}+\mathbf{R}_{m}^{i j} \mathbf{R}_{m}^{j i}\right)^{*} \mathbf{J}\right] .
\end{aligned}
$$

3.2. Modified Spatial Difference Smoothing Algorithm. The spatial difference smoothing algorithm is proposed by [20] using the Toeplitz decomposition characteristics and differential smoothing operations of the uniform linear array covariance matrix to estimate the target angle. The expression of the spatial difference matrix is as follows:

$$
\Delta \mathbf{R} \triangleq \mathbf{R}-\mathbf{J R}^{*} \mathbf{J}
$$

If formula (7) is replaced by formula (14), we can obtain

$$
\Delta \mathbf{R}=\mathbf{R}_{\mathrm{c}}-\mathbf{R}_{\mathrm{nc}}+\boldsymbol{\varphi}-\mathbf{J}\left(\mathbf{R}_{\mathrm{c}}-\mathbf{R}_{\mathrm{nc}}+\boldsymbol{\varphi}\right)^{*} \mathbf{J},
$$

where $\mathbf{R}_{\mathrm{nc}}$ and $\boldsymbol{\varphi}$ are Hermite, Toeplitz matrix, conforming to conjugate reverse order invariance:

$$
\begin{aligned}
\mathbf{R}_{\mathrm{nc}} & =\mathbf{J} \mathbf{R}_{\mathrm{nd}}^{*} \mathbf{J} \\
\boldsymbol{\varphi} & =\mathbf{J} \varphi^{*} \mathbf{J} .
\end{aligned}
$$

So, formula (15) can be written as follows:

$$
\Delta \mathbf{R} \triangleq \mathbf{R}_{\mathrm{c}}-\mathbf{J R}_{\mathrm{c}}^{*} \mathbf{J} .
$$

Formula (17) shows that there is neither the noise item nor the Toeplitz term of the independent source, which contains only the information of coherent sources.

From the construction process of matrix $\Delta \mathbf{R}$, it can be seen that $\Delta \mathbf{R}$ is a negative antisymmetric matrix, and its eigenvalues are the combination of positive and negative. Since the trace of a matrix equals the sum of eigenvalues, when the order $K$ of $\Delta \mathbf{R}$ is an odd number, it must have a 0 eigenvalue as follows:

$$
\operatorname{rank}(\Delta \mathbf{R})=K-1 .
$$

It can be seen that the negative antisymmetry of $\Delta \mathbf{R}$ will lead to rank deficit in some cases, and $\Delta \mathbf{R}$ needs to be corrected and decoherent.

In this paper, an improved spatial difference smoothing (ISDS) algorithm is proposed, which preprocesses $\Delta \mathbf{R}$ by Formulae (12) and (13):

$$
\begin{aligned}
\Delta \widetilde{\mathbf{R}}_{\mathrm{f}}= & \frac{1}{2 q} \sum_{i=1}^{q} \sum_{j=1}^{q}\left(\Delta \mathbf{R}_{m}^{i i} \Delta \mathbf{R}_{m}^{j j}+\Delta \mathbf{R}_{m}^{i j} \Delta \mathbf{R}_{m}^{j i}\right), \\
\Delta \widetilde{\mathbf{R}}_{\mathrm{fb}}= & \frac{1}{4 q} \sum_{i=1}^{q} \sum_{j=1}^{q}\left[\left(\Delta \mathbf{R}_{m}^{i i} \Delta \mathbf{R}_{m}^{j j}+\Delta \mathbf{R}_{m}^{i j} \Delta \mathbf{R}_{m}^{j i}\right)\right. \\
& \left.+\mathbf{J}\left(\Delta \mathbf{R}_{m}^{i i} \Delta \mathbf{R}_{m}^{j j}+\Delta \mathbf{R}_{m}^{i j} \Delta \mathbf{R}_{m}^{j i}\right)^{*} \mathbf{J}\right],
\end{aligned}
$$

where $\Delta \mathbf{R}_{m}^{i i}=\Delta \mathbf{R}(i: I+i-q, i: I+i-q)$ and $\Delta \mathbf{R}_{m}^{i j}=\Delta \mathbf{R}$ $(i: I+i-q, j: I+j-q)$.

In order to further distinguish the proposed ISDS algorithm, the operation of formula (18) is called the improved forward spatial difference smoothing (IFSDS) algorithm in this paper, and the operation of formula (19) is called the improved forward and backward spatial difference smoothing (IFBSDS) algorithm. 
3.3. The Principle of MWF-ISDS Algorithm. When the number of virtual elements is large, the computation of signal subspace directly through eigenvalue decomposition is large. In this paper, an improved spatial difference smoothing algorithm based on multistage Wiener filtering is proposed. The signal subspace is obtained by the decomposition process of multistage Wiener filtering, and the computational complexity of the algorithm is further reduced. The processing flow is shown in Figure 2.

In Figure 2, $x_{0}$ is the initial array antenna receiving data vector; $x_{1}, \ldots$ is each stage residual vector after the recursive projection of the array antenna receiving data vector; $h_{1}, h_{2}$, $\ldots$ is the next recursive normalized projection vector; $C_{1}, C_{2}$, $\ldots$ is each stage forward recursive vector of the array antenna received data vector; and $\mathbf{d}=\left[\mathbf{d}_{1}, \mathbf{d}_{2}, \ldots, \mathbf{d}_{K}\right]^{\mathrm{T}}$ is oriented vector space, which can be expressed in the following way:

$$
\mathbf{d}=\left[\mathbf{d}_{1}, \mathbf{d}_{2}, \ldots, \mathbf{d}_{K}\right]^{\mathrm{T}}=\left[\begin{array}{c}
\mathbf{h}_{1} \\
\mathbf{B}_{1} \mathbf{h}_{2} \\
\vdots \\
\prod_{r=1}^{K-2} \mathbf{B}_{r} \mathbf{h}_{K-1} \\
\prod_{r=1}^{K-1} \mathbf{B}_{r} \mathbf{h}_{K}
\end{array}\right] \cdot \mathbf{x}_{0}(t),
$$

where $K$ represents the number of filter recursion times.

If vector $\left[w_{1}, w_{2}, \ldots, w_{\mathrm{I}}\right]=\left[\mathbf{h}_{1}, \mathbf{B}_{1} \mathbf{h}_{2}, \ldots, \prod_{r=1}^{K-2} \mathbf{B}_{r} \mathbf{h}_{K-1}\right.$, $\left.\prod_{r=1}^{K-1} \mathbf{B}_{r} \mathbf{h}_{K}\right]$ is used to represent the forward recursive matching filter of multistage Wiener filter, it can be proved that [18]

$$
\operatorname{span}\left\{w_{1}, w_{2}, \ldots, w_{\mathrm{I}}\right\}=\operatorname{span}\left\{\mathbf{h}_{1}, \mathbf{R}_{\mathrm{r}} \mathbf{h}_{1}, \mathbf{R}_{\mathrm{r}}^{2} \mathbf{h}_{1}, \ldots, \mathbf{R}_{\mathrm{r}}^{I-1} \mathbf{h}_{1}\right\},
$$

where $\mathbf{R}_{\mathrm{r}}$ represents the received data covariance matrix of MIMO radar and $I$ is the number of virtual array elements.

The proposed ISDS algorithm is used to decompose the matrix $\Delta \widetilde{\mathbf{R}}_{\mathrm{f}}$ or $\Delta \widetilde{\mathbf{R}}_{\mathrm{fb}}$ to get the subspace $\mathbf{U}_{\mathrm{S}}=\left[\mathbf{u}_{1}, \mathbf{u}_{2}, \ldots\right.$, $\left.\mathbf{u}_{\mathrm{G}}\right]$. The relationship between the signal subspace $\mathbf{V}_{\mathrm{S}}=$ $\left[\mathbf{v}_{1}, \mathbf{v}_{2}, \ldots, \mathbf{v}_{\mathrm{G}}\right]$ and $\mathbf{U}_{\mathrm{S}}$ of MWF algorithm satisfies [21]

$$
\operatorname{span}\left\{\mathbf{v}_{1}, \mathbf{v}_{2}, \ldots, \mathbf{v}_{\mathrm{G}}\right\}=\operatorname{span}\left\{\mathbf{u}_{1}, \mathbf{u}_{2}, \ldots, \mathbf{u}_{\mathrm{G}}\right\} .
$$

The initialization reference signal of the MWF algorithm is the row mean of $\Delta \widetilde{\mathbf{R}}$ as follows:

$$
d_{0}(t)=\frac{1}{I} \sum_{i=1}^{I} \Delta \widetilde{\mathbf{R}}(i,:) .
$$

By $G$ recursions, the subspace of MIMO radar echo data can be obtained as

$$
\mathbf{U}_{\mathrm{S}}=\left[w_{1}, w_{2}, \ldots, w_{\mathrm{G}}\right] .
$$

The noise subspace of MIMO radar echo data is

$$
\mathbf{U}_{\mathrm{N}} \mathbf{U}_{\mathrm{N}}^{\mathrm{H}}=\mathbf{I}-\mathbf{U}_{\mathrm{S}} \mathbf{U}_{\mathrm{S}}^{\mathrm{H}} \text {. }
$$

According to the MUSIC algorithm, the DOAs for $G$ coherent sources are

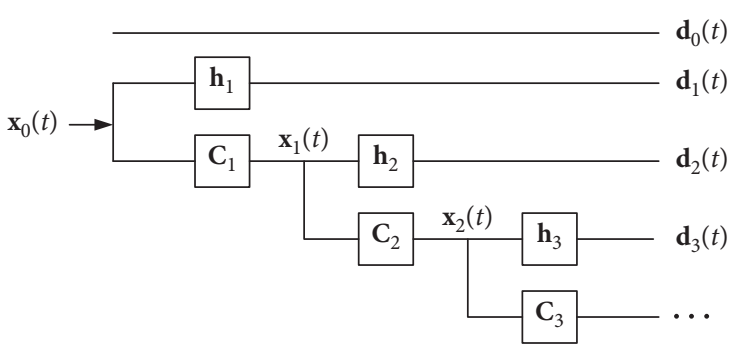

FIgURE 2: Flow-process diagram of multistage Wiener filter.

$$
\widehat{\theta}=\arg \min _{\theta}\left\{\mathbf{b}^{\mathrm{H}}(\theta) \mathbf{U}_{\mathrm{N}} \mathbf{U}_{\mathrm{N}}^{\mathrm{H}} \mathbf{b}(\theta)\right\}
$$

Based on the above analysis, the operational steps of angle estimation by using the MWF-ISDS algorithm can be summarized as follows:

Step 1. According to formula (6), the received signal of matched filtering is dimensionality reduced

Step 2. Use the MWF algorithm to estimate the angle of independent sources

Step 3. According to formula (15), the array difference matrix $\Delta \mathbf{R}$ of MIMO radar is calculated

Step 4. Calculate $\Delta \widetilde{\mathbf{R}}_{\mathrm{f}}$ or $\Delta \widetilde{\mathbf{R}}_{\mathrm{fb}}$ according to formula (19) or (20)

Step 5. Use the MWF algorithm to estimate the signal subspace of $\Delta \widetilde{\mathbf{R}}_{\mathrm{f}}$ or $\Delta \widetilde{\mathbf{R}}_{\mathrm{fb}}$

Step 6. Calculate the noise subspace according to formula (26)

Step 7. Estimate the angle of the coherent sources using formula (27)

\section{Performance Analysis of MWF- ISDS Algorithm}

4.1. Improved Performance of the Algorithm. In order to compare the algorithm performance, the improvement of the signal-to-noise ratio can be expressed by the ratio of covariance matrix trace of source to covariance matrix trace of noise. Then, the SNR improvement factor $Q_{\text {sn }}$ is defined as follows:

$$
Q_{\mathrm{sn}}=\frac{\operatorname{tr}(\mathbf{P})}{\operatorname{tr}(\varphi)}
$$

where $\operatorname{tr}(\mathbf{P})$ is the trace of the source covariance matrix and $\operatorname{tr}(\varphi)$ is the trace of the noise covariance matrix. The larger the value $Q_{s n}$ is, the smaller the noise perturbation is, and the better the estimation performance of the algorithm is.

If the classic forward smoothing, there is 


$$
\begin{aligned}
\mathbf{R}_{\mathrm{f}}=\frac{1}{q} \sum_{i=1}^{q} \mathbf{R}_{m}^{i i} & =\mathbf{B}_{m}\left(\frac{1}{q} \sum_{i=1}^{q} \mathbf{D}^{i-1} \mathbf{P}\left(\mathbf{D}^{i-1}\right)^{\mathrm{H}}\right) \mathbf{B}_{m}^{\mathrm{H}}+\sigma^{2} \mathbf{I}_{m} \\
& =\mathbf{B}_{m} \mathbf{P}_{\mathrm{f}} \mathbf{B}_{m}^{\mathrm{H}}+\sigma^{2} \mathbf{I}_{m},
\end{aligned}
$$

where $\mathbf{P}_{\mathrm{f}}$ is the echo covariance matrix of the classical forward smoothing algorithm.
By formula (29), the signal-to-noise ratio improvement factor of the classical forward smoothing algorithm can be obtained as follows:

$$
Q_{\mathrm{sn}-\mathrm{f}}=\frac{\operatorname{tr}\left(\mathbf{P}_{\mathrm{f}}\right)}{\operatorname{tr}\left(\sigma^{2} \mathbf{I}_{m}\right)} .
$$

If the improved forward smoothing, there is

$$
\widetilde{\mathbf{R}}_{\mathrm{f}}=\frac{1}{2 q} \mathbf{B}_{m}\left(\left(\sum_{\substack{i, j=1 \\ i \neq j}}^{q} w_{i j}+2 \sigma^{2}\right)\left(\sum_{\substack{i, j=1 \\ i \neq j}}^{q} \mathbf{D}^{i-1} \mathbf{P}\left(\mathbf{D}^{j-1}\right)^{\mathrm{H}}\right)+2\left(\sum_{i=1}^{q} w_{i}+\sigma^{2}\right)\left(\sum_{i=1}^{q} \mathbf{D}^{i-1} \mathbf{P}\left(\mathbf{D}^{i-1}\right)^{\mathrm{H}}\right) \mathbf{B}_{m}^{\mathrm{H}}+\sigma^{4} \mathbf{I}_{m},\right.
$$

where $w_{i j}=\boldsymbol{\beta}^{\mathrm{H}}\left(\mathbf{D}^{i-1}\right)^{\mathrm{H}} \mathbf{B}_{m}^{\mathrm{H}} \mathbf{B}_{m} \mathbf{D}^{j-1} \boldsymbol{\beta}$ and $w_{j}=\boldsymbol{\beta}^{\mathrm{H}}\left(\mathbf{D}^{j-1}\right)^{\mathrm{H}}$ $\mathbf{B}_{m}^{\mathrm{H}} \mathbf{B}_{m} \mathbf{D}^{j-1} \boldsymbol{\beta}$.
By formula (31), the improvement factor of the signal to noise ratio of the improved forward smoothing algorithm can be obtained as follows:

$$
\widetilde{Q}_{\mathrm{sn}-\mathrm{f}}=\frac{\operatorname{tr}\left((1 / 2 q)\left(\sum_{i, j=1, i \neq j}^{q} w_{i j}+2 \sigma^{2}\right)\left(\sum_{i, j=1, i \neq j}^{q} \mathbf{D}^{i-1} \mathbf{P}\left(\mathbf{D}^{j-1}\right)^{\mathrm{H}}\right)\right)}{\operatorname{tr}\left(\sigma^{4} \mathbf{I}\right)}+\left(\frac{1}{\sigma^{2}} \sum_{j=1}^{q} w_{j}+1\right) Q_{\mathrm{sn}-\mathrm{f} .} .
$$

It is clear that the proposed forward smoothing algorithm is better than the classical forward smoothing algorithm.

4.2. Comparison of Computational Complexity. If do not reduce the covariance matrix dimension, the multiplication times of the subspace algorithms as MUSIC approximate to $O\left\{M^{2} N^{2} L\right\}+O\left\{M^{3} N^{3}\right\}$. The number of multiplication times required by the multistage Wiener filtering method is approximately [22] $O\{G M N L\}$. If the dimension is reduced, the subspace algorithms such as MUSIC need to decompose the $(M+N-1)$ covariance matrix, and the multiplication times is $O\left\{(M+N-1)^{2} L\right\}+O\left\{(M+N-1)^{3}\right\}$. The number of multiplication times required by the multistage Wiener filtering method is approximately $O\{G(M+$ $N-1) L\}$. It can be seen that the MWF-ISDS algorithm proposed in this paper reduces the dimension of the matrix operation by reducing the dimension of the dimension, and further reduces the computational complexity by using the multistage Wiener filtering method.

\section{Computer Simulation Results}

It is assumed that the number of transmitter elements and the number of receiving elements of the monostatic MIMO radar are 6 and 8, respectively, and the number of virtual elements is 13 after dimensionality reduction. It may be possible to establish a spatial stationary color covariance matrix of $\varphi$, and its $k$ th line $l$ th column element is $[\varphi]_{k, l}=\sigma_{n}^{2} 0.9^{|k-l|} \exp \{j \pi(k-l) / 16\}$. The signal-to-noise ratio is $S / N=10 \lg \left(\sigma_{s}^{2} / \sigma_{n}^{2}\right)$, where $\sigma_{s}^{2}$ indicates signal power and $\sigma_{n}^{2}$ indicates noise power.

Experiment 1 (comparison of spatial spectrum curves of different algorithms under colored noise mixed source background). In the background of colored noise, angle estimation of 6 equal power mixed sources is carried out. The angles are $-40^{\circ},-30^{\circ},-10^{\circ}, 5^{\circ}, 25^{\circ}$, and $35^{\circ}$, respectively. The first 4 are coherent sources and the other 2 are incoherent sources. The number of pulses is 200 , the number of smooth subarrays is 4 , and the signal-to-noise ratio is fixed to $0 \mathrm{~dB}$. Figure 3 simulates the normalized spatial spectral curves of six 


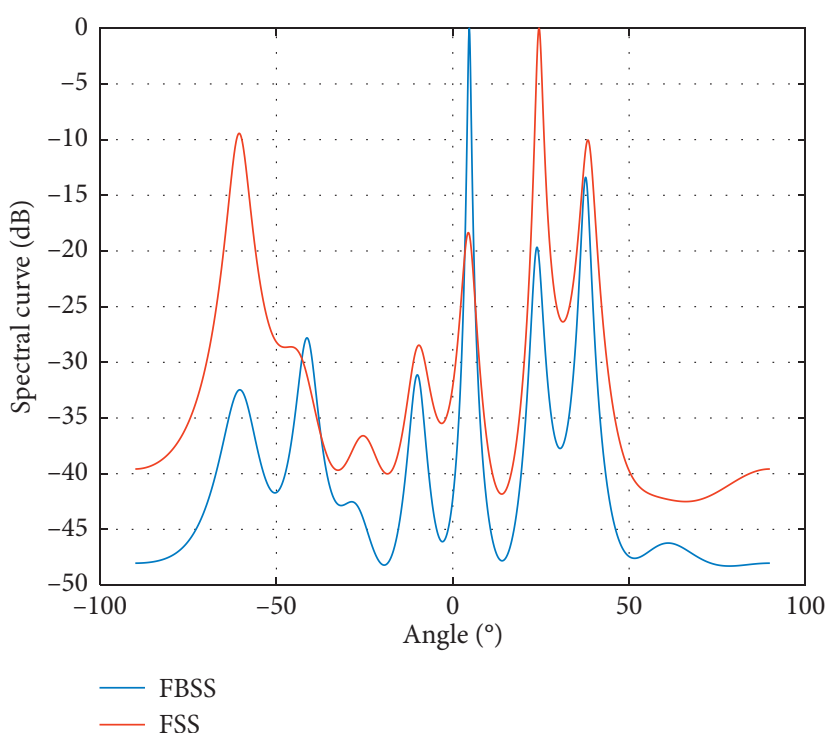

(a)

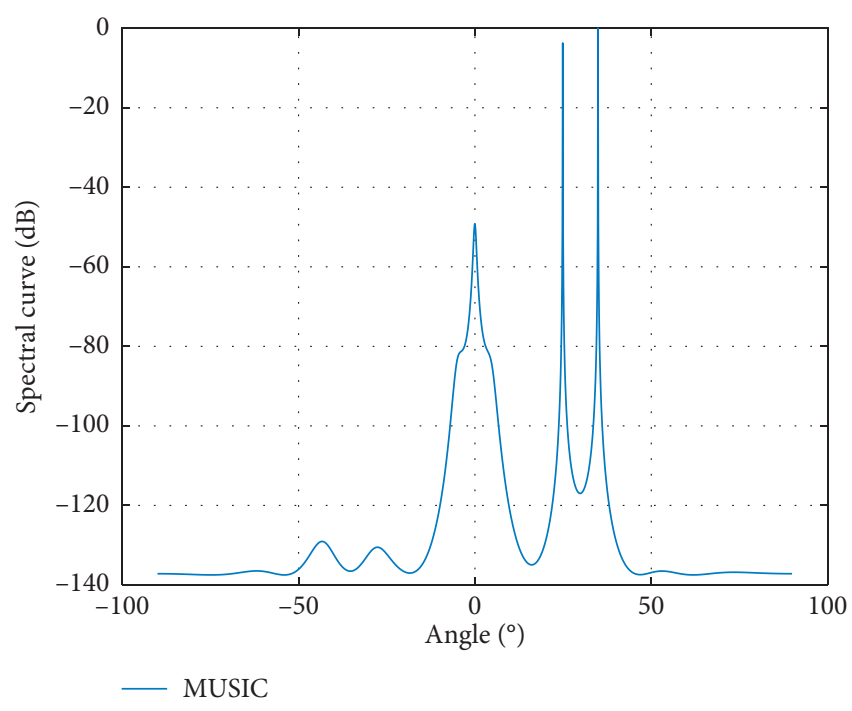

(b)

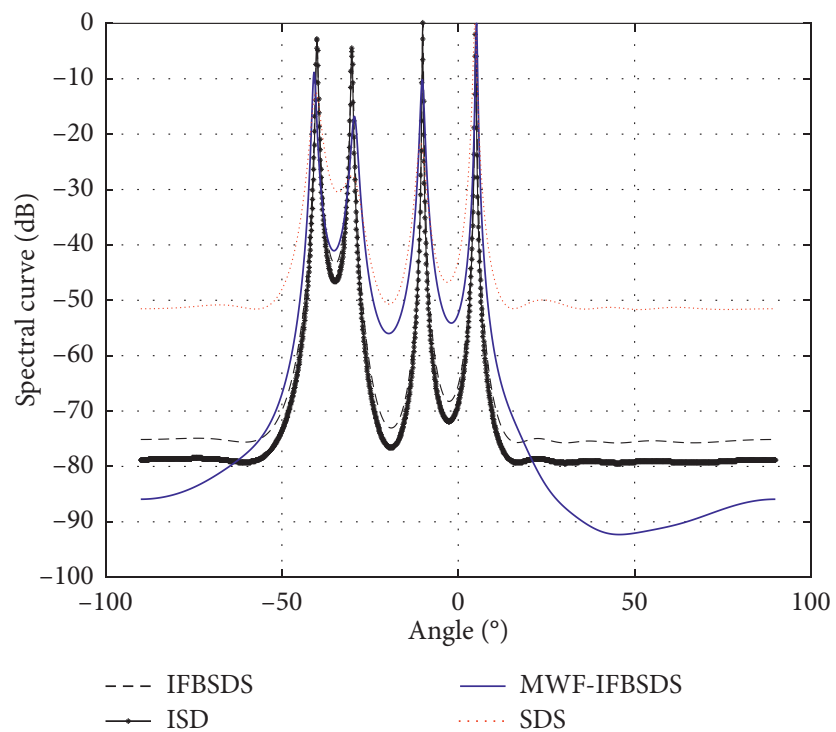

(c)

FIGURE 3: Angle estimation performance of mixed noise sources using different algorithms. (a) FSS and FBSS algorithm. (b) MUSIC algorithm. (c) SDS, IFBSDS, ISD, and MWF-IFBSDS algorithms.

different algorithms of FSS, FBSS, MUSIC, SDS, IFBSDS, and MWF-IFBSDS.

The simulation results from Figure 3 show that, under the background of color noise, the FSS and FBSS algorithms only use the smooth processing without differential processing; the angle estimation performance is low, and the estimated results have a large error with the true direction of arrival. The MUSIC algorithm cannot estimate the angle of the coherent source and can effectively estimate the angle of the independent source. But because of the inability to overcome the influence of colored noise, the normalized spatial spectral curve still has a large pseudo peak. The SDS, IFBSDS, and MWF-IFBSDS algorithms use both the smoothing and the differential processing, so it is good to estimate the angle of the coherent source in the background of the color noise. Because of the improved smoothing algorithm in ISD, IFBSDS, and MWF-IFBSDS algorithms, the estimation performance of the angle is better than that of the SDS algorithm. Compared with the ISD algorithm, the proposed MWF-IFBSDS algorithm still maintains the angle estimation performance which is not much different from the ISD algorithm with reducing the computational complexity.

Experiment 2 (statistical performance comparison of angle estimation algorithms under colored noise coherent sources). In the background of colored noise, the angle estimation of 2 equal power coherent sources is $0^{\circ}$ and $10^{\circ}$, respectively. The number of pulsing pulses is 200 and the number of smooth subarrays is 2 . Figure 4 simulates angle 


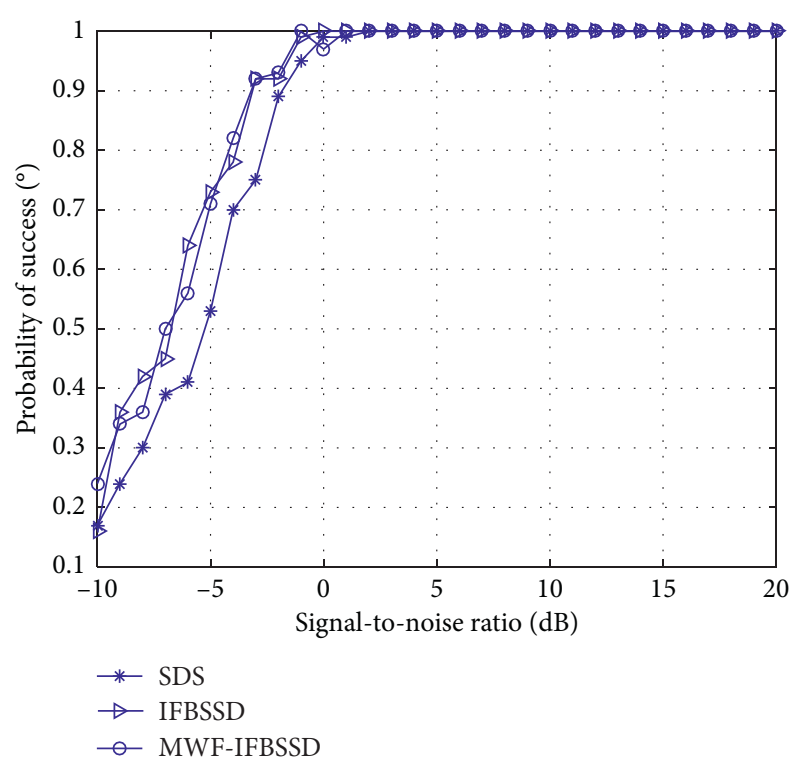

(a)

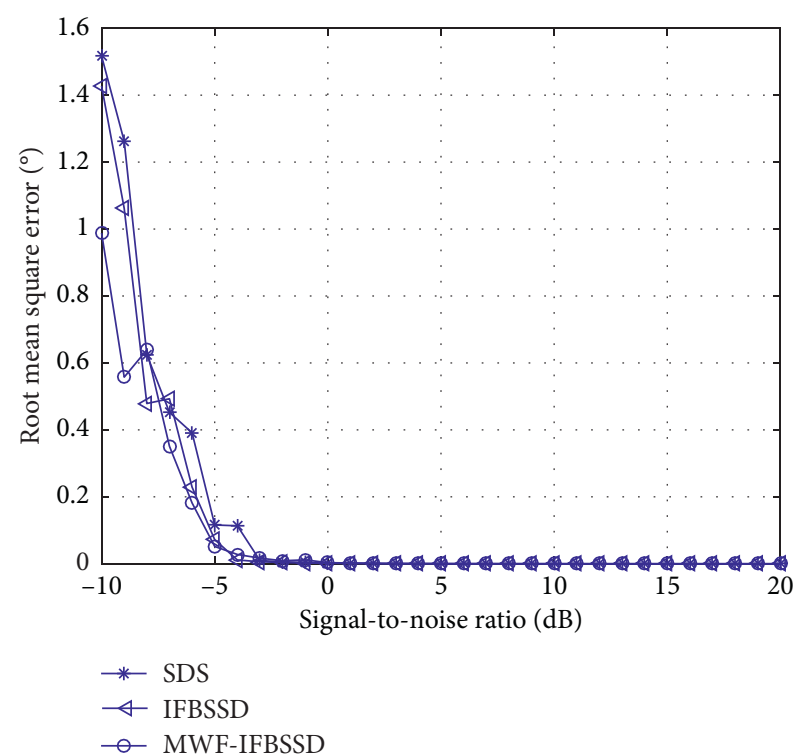

(b)

FIGURE 4: Comparison of angle estimation performance when SNR varies. (a) Success probability. (b) Root mean square error.

estimation performance of SDS, IFBSDS, and MWF-IFBSDS algorithms in 200 Monte-Carlo experiments.

The simulation results in Figure 4 show that when the SNR is low, the IFBSDS and MWF-IFBSDS algorithms outperform the SDS algorithm in terms of probability of success and root mean square error of estimation. In the case of low SNR, the angle estimation performance of the IFBSDS and MWFIFBSDS algorithms is better than that of the SDS algorithm. With the increase of SNR, the statistical performance of the three algorithms is basically the same.

\section{Conclusions}

In this paper, the echo model under the background of colored noise is established and the characteristics of its covariance matrix are analyzed. An ISDS (MWF-ISDS) angle estimation algorithm based on multistage Wiener filtering is proposed. The improved performance and computational complexity of the algorithm are analyzed and simulated. Theoretical analysis and simulation experiments show that the MWF-IFBSDS algorithm has better angle estimation performance than the FSS, FBSS, MUSIC, and ISDS algorithms under low SNR and small angle interval, and the proposed algorithm has stronger array element saving ability and source overload capability.

\section{Data Availability}

The simulation data of program used to support the findings of this study are available from the corresponding author upon request.

\section{Conflicts of Interest}

The authors declare that they have no conflicts of interest.

\section{Authors' Contributions}

Jian Gong and Yiduo Guo designed the algorithm scheme. Jian Gong performed the experiments and analyzed the experiment results. Yiduo Guo contributed to the manuscript drafting and critical revision. All authors read and approved the final manuscript.

\section{Acknowledgments}

This work was supported by the National Natural Science Foundation of China under Grant nos. 61601502 and 61501501.

\section{References}

[1] G. Zheng, "DOA estimation in MIMO radar with non-perfectly orthogonal waveforms," IEEE Communications Letters, vol. 21, no. 2, pp. 414-417, 2017.

[2] M. Yang, B. Chen, G. Zheng et al., "Reduced-dimensional unitary ESPRIT algorithm for monostatic MIMO radar," in Proceedings of 2011 IEEE CIE International Conference on Radar, vol. 36, no. 6, pp. 1062-1067, Chengdu, China, October 2011.

[3] C. Dang, W. Zhang, and Y. Hu, "DOA estimation in subarraybased MIMO radar with distributed inter-subarray calibration," Journal of North University of China, vol. 38, no. 2, pp. 217-220, 2017.

[4] K. Duan, M. Liu, H. Dai, F. Xu, and W. Liu, "A two-stage detector for mismatched subspace signals," IEEE Geoscience and Remote Sensing Letters, vol. 14, no. 12, pp. 2270-2274, 2017.

[5] J. Shi, G. Hu, and T. Lei, "DOA estimation algorithms for lowangle targets with MIMO radar," Electronics Letters, vol. 52, no. 8, pp. 652-654, 2016.

[6] D. Wang and J. Guo, "A method for DOA estimation of coherent signals in MIMO radar," Modern Defence Technology, vol. 45, no. 1, pp. 194-198, 2017. 
[7] C. Wang, L. Gong, and S. Wu, "Joint DOD and DOA estimation for bistatic MIMO radar in the presence of colored noise," Systems Engineering and Electronics, vol. 37, no. 10, pp. 2255-2259, 2015.

[8] W. Wang, X. Wang, and L. Xin, "An angle estimation algorithm for MIMO radar in the presence of colored noise fields," in Proceedings of the IEEE CIE International Conference On Radar, pp. 1097-1105, Chengdu, China, October 2011.

[9] H. Chen, C. Hou, W. Liu, W.-P. Zhu, and M. N. S. Swamy, "Efficient two-dimensional direction-of-arrival estimation for a mixture of circular and noncircular sources," IEEE Sensors Journal, vol. 16, no. 8, pp. 2527-2536, 2016.

[10] S. Wang, Q. He, Z. He et al., "Waveform design for MIMO over-the-horizon radar detection in signal dependent clutter and colored noise," in Proceeding of the IET International Radar Conference, pp. 14-16, Xi'an, China, 2013.

[11] P. Wang, T. Qiu, J. Li et al., "Estimation of joint multi-parameters of near-field sources based on the fourth-order cumulant," Journal of Dalian University of Technology, vol. 55, no. 6, pp. 625-631, 2015.

[12] W.-b. Fu, T. Su, Y.-b. Zhao et al., "Joint estimation of angle and doppler frequency for bistatic MIMO radar in spatial colored noise based on temporal-spatial structure," Journal of Electronics \& Information Technology, vol. 33, no. 7, pp. 1649-1654, 2011.

[13] Z. He, J. Shi, and H. Xu, "Multitarget localization for bistatic MIMO radar in Gaussian colored noise," Journal of Detection \& Control, vol. 38, no. 6, pp. 68-73, 2016.

[14] X. Li, Z. Zhang, T. Wang et al., "Target rapid location of coherent target for bistatic MIMO radar," Journal of Signal Processing, vol. 32, no. 3, pp. 370-377, 2016.

[15] X. Ma, X. Dong, and Y. Xie, "An improved spatial differencing method for DOA estimation with the coexistence of uncorrelated and coherent signals," IEEE Sensors Journal, vol. 16, no. 10, pp. 3719-3723, 2016.

[16] J. Shi, G. Hu, F. Sun, B. Zong, and X. Wang, "Improved spatial differencing scheme for 2-D DOA estimation of coherent signals with uniform rectangular arrays," Sensors, vol. 17, no. 9, p. 1956, 2017.

[17] J. Gong, H. Wang, and Y. Guo, "An angle estimation method for monostatic MIMO radar based on RCC-FLOM algorithm," Wireless Communications and Mobile Computing, vol. 2019, Article ID 9710656, 7 pages, 2019.

[18] X. Zhang, G. Zhang, J. Li et al., Target Location of MIMO radar, National Defense Industry Press, Beijing, China, 2014.

[19] A. B. Gershman, A. L. Matveyev, and J. F. Bohme, "Maximum-likelihood estimation of signal power in sensor array in the presence of unknown noise field," IEE Proceedings-Radar, Sonar and Navigation, vol. 42, no. 10, pp. 218-224, 1995.

[20] C. Qi, Study on Robustness of High Resolution Wave of Arrival estimation, Air Force Engineering University, Xi'an, China, 2005.

[21] M. L. Honig and W. M. Weimin Xiao, "Performance of reduced-rank linear interference suppression," IEEE Transactions on Information Theory, vol. 47, no. 5, pp. 1928-1946, 2001.

[22] L. Huang, Study on Fast Subspace Estimation Method and its Application to Array Signal processing, Xidian University, Xi'an, China, 2005. 


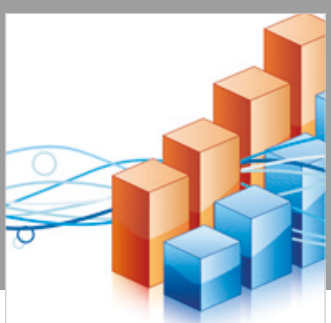

Advances in

Operations Research

\section{-n-m}
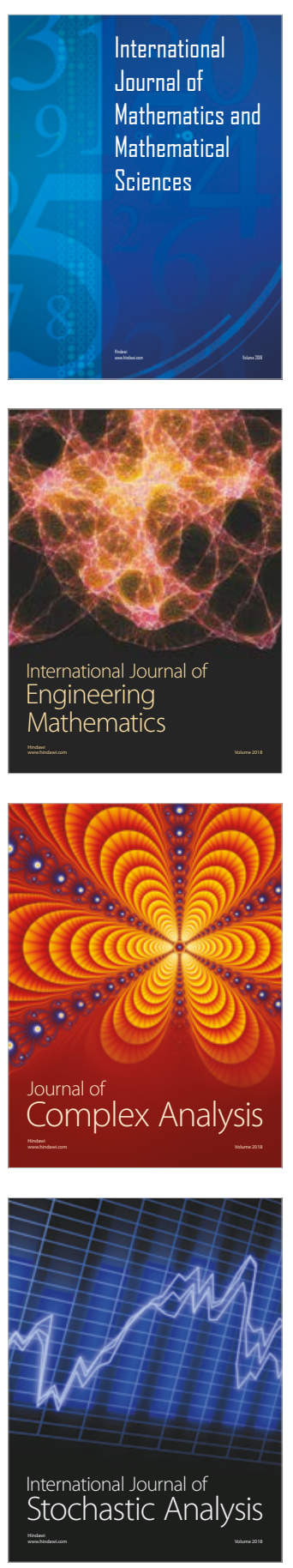
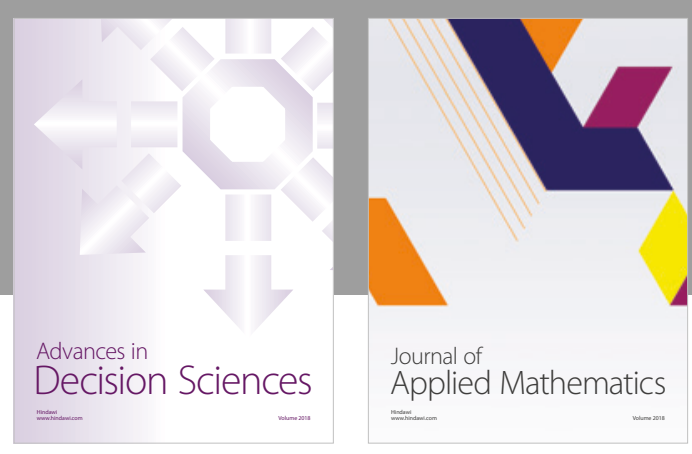

Journal of

Applied Mathematics
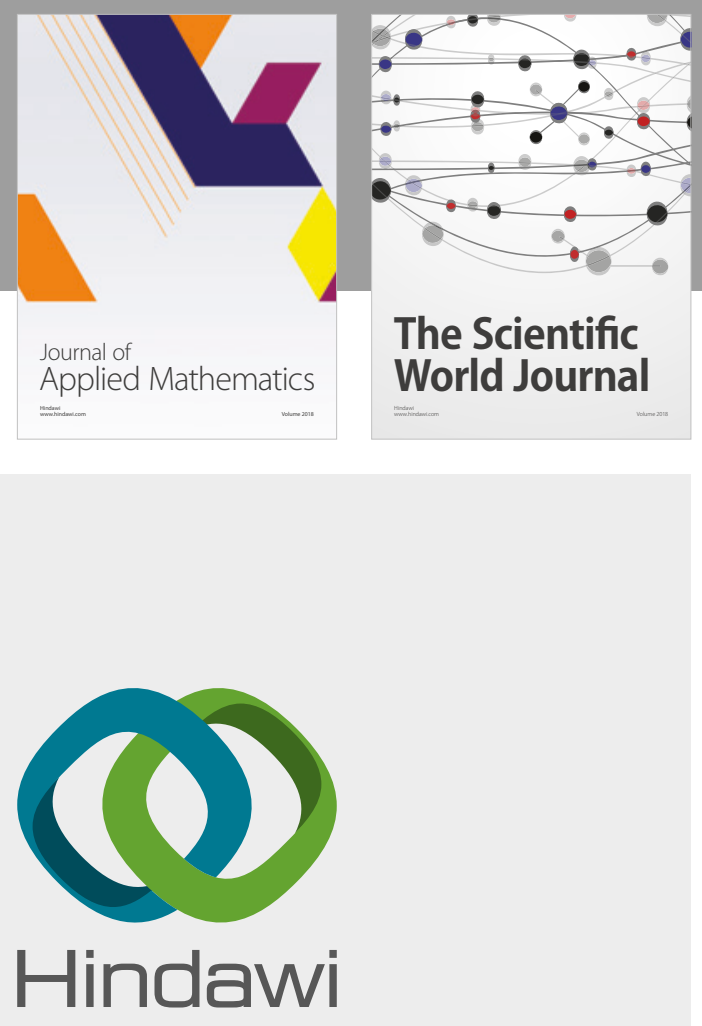

Submit your manuscripts at

www.hindawi.com

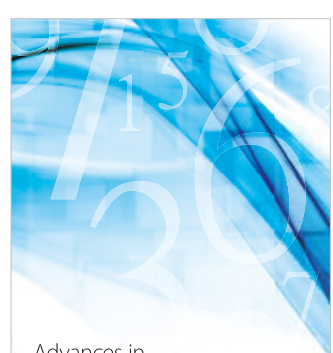

Advances in
Numerical Analysis
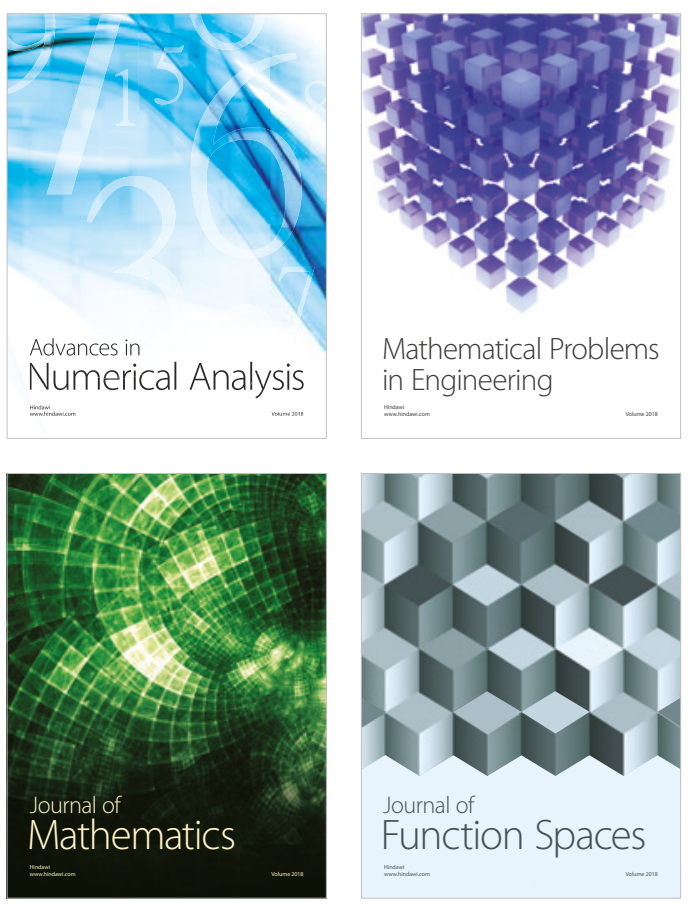

Mathematical Problems in Engineering

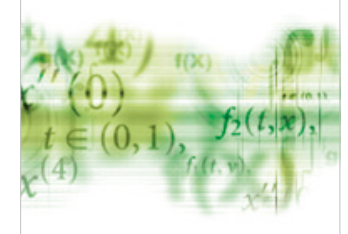

International Journal of

Differential Equations

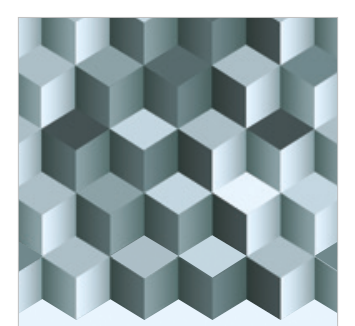

Journal of

Function Spaces

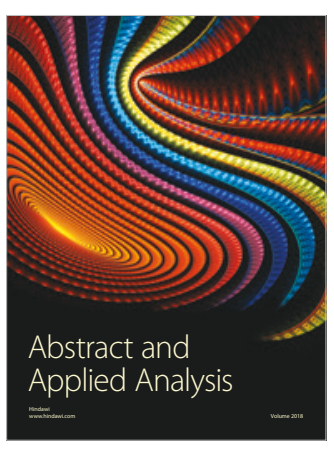

The Scientific

World Journal

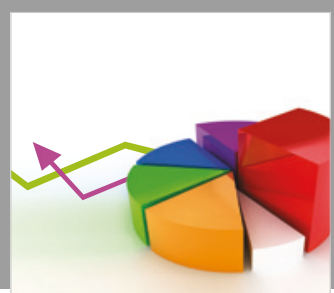

Journal of

Probability and Statistics
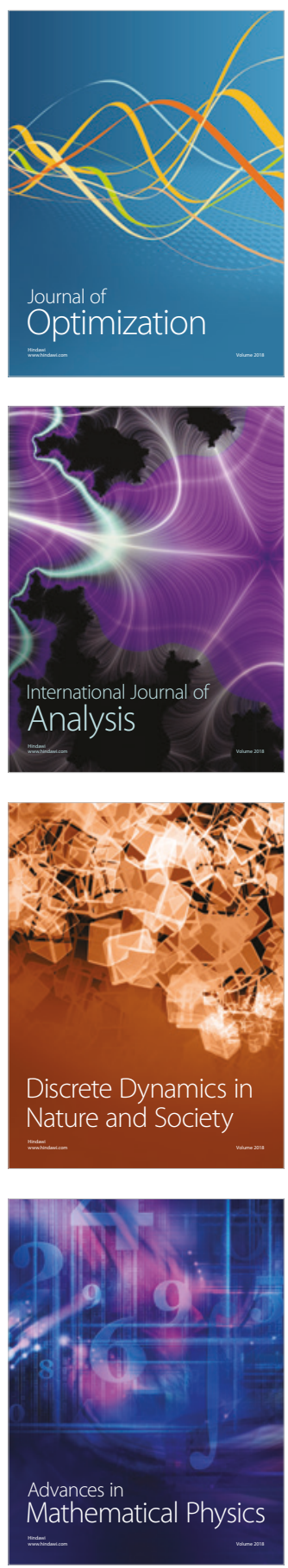